\title{
Pitie et Non-violence dans 1S 24-26 : Cas de David Versus Saül et Nabal
}

\author{
Augustin L. Kagunge (Katholieke Universiteit LeUVen)
}

\begin{abstract}
This article takes root in the reading of the narrative sequence which we find 1S 24-26 where three narratives follow one after another: at first to David who leads Saul to Engaddi, then to Abigail who diverts David from his plan to avenge Nabal, and finally, to David who once more saves the life of Saul in the desert of Ziph. To emphasize the need for pity and nonviolence in the society, it seems interesting to us to raise the context antecedent to this sequence where the hostility of Saul towards David leaves no shadow of a doubt. Indeed, devoured by jealousy in the face of David's successes, Saul tries with a doggedness to eliminate the latter who dashes off desperately until golden opportunities are presented to him to eliminate Saul. He does not eliminate him, but he gets only the piece of his coat as he surprises him while he was covering his feet. Then, for a second time, he still saves his life when he perceives him sleeping near Abner. Refusing to hurt him, he takes the lance which was at his bedside as well as the gourd of water. Pity and non-violence towards enemies practised by David could be the resources which our society needs to strengthen to consolidate conviviality. In this context where wars, conflicts, hatred, vengeances and terrorism seem to gain ground, could the rediscovery of these two values be a challenge to reveal for our world?
\end{abstract}

KEYWORDS: Pitié, non-vengeance, non-violence, réconciliation, fraternité

\section{A INTRODUCTION}

Les chapitres 24 à 26 du premier livre de Samuel constituent ce que l'on appelle une séquence narrative ${ }^{1}$, car ils sont formés d'une trame unifiée ${ }^{2}$ où des épisodes

\footnotetext{
* Submitted: 25/08/2018; peer-reviewed: 04/02/2019; accepted: 14/03/2019. Augustin L. Kagunge, "Pitie et Non-violence dans 1S 24-26 : Cas de David Versus Saül et Nabal," Old Testament Essays 32 no. 1 (2019): 155-173. DOI: https://doi.org/ 10.17159/2312-3621/2019/v32n1a9.

1 Une séquence narrative est « une unité narrative composée de plusieurs microrécits, articulés l'un à l'autre par un thème commun ou reliés par la présence d'un même personnage central », cf. Daniel Marguerat \& Yvan Bourquin, Pour Lire les Récits Bibliques : Initiation à l'analyse narrative. $4^{\text {ème }}$ éd. (Paris-Genève-Montréal : CerfLabor et Fides-Novalis, 2009), 47.

2 Une lecture attentive montre que 1S 24-26 constitue une séquence narrative. On y retrouve une unité thématique. Comme l'écrit Jacques Vermeylen, “David le Non-
} 
se succèdent les uns aux autres. Chaque épisode porte en lui-même un sens et revêt une certaine importance aussi bien par rapport à lui-même que par rapport à toute la séquence. Nous nous focalisons, dans cette analyse, sur les trois épisodes situés en $1 \mathrm{~S} 24-26^{3}$ à savoir d'une part, les deux récits où le narrateur raconte la manière dont David épargne la vie à Saül à Engaddi et au désert de Ziph, et d'autre part, le récit de Nabal et de sa femme Abigayil où David reçoit de cette dernière une leçon de non-violence. Par le biais de l'analyse narrative, nous investiguerons les effets de sens que ces récits produisent sur le lecteur. Pour cela, nous serons particulièrement attentifs à la manière dont le texte communique avec le lecteur et comment il fait sens auprès de ce dernier. Nous envisageons ici le lecteur implicite qui, selon Daniel Marguerat et Yvan Bourquin, est le « récepteur du récit construit par le texte et apte à en actualiser les significations dans la perspective induite par l'auteur. ${ }^{4}$

Puisque ces récits succèdent à d'autres, sans lesquels ils ne peuvent être correctement compris, qu'il nous soit permis de revenir jusqu'au chapitre 16. Saül est le premier roi d'Israël. À la suite de sa désobéissance à YHWH $(1 \mathrm{~S} 15,9)$,

Violent,' In La Bible en Récits. L'Exégèse Biblique à l'Heure du Lecteur (ed. Daniel Marguerat ; Genève : Labor et Fides, 2003), 136, ces trois chapitres reflètent un schéma commun : il est question, dans chacun d'eux, d'un homme puissant et méchant auquel David, le héros, est confronté : d'un côté, Saül le pourchasse en 1S 24 et 26 ; de l'autre, Nabal refuse de lui apporter assistance en $1 \mathrm{~S} 25$. Par la suite, le récit montre la manière dont il fait preuve de non-violence vis-à-vis de Saül et de Nabal qu'il tient pourtant à sa merci. Aussi, des indices littéraires (cf. Patrick D. Miller \& J. J. M. Robert, The Hand of the Lord : A Reassessment of the "Ark Narrative", of 1 Samuel (Baltimore: Johns Hopkins University Press, 1977), 19-23) tels qu'une forte récurrence des particules ואתה (dont le tiers est concentré en 1S 24-26) et הנה (reprise dix fois sur les 48 emplois répertoriés dans $1 \mathrm{~S}$ ), confèrent à ces trois chapitres une cohérence interne. Pour plus de détails, on peut lire Robert Polzin, Samuel and the Deuteronomist. A Literary Study of the Deuteronomic History. Part II, 1 Samuel (BloomingtonIndianapolis: Indiana University Press, 1989), 205.

3 Nous utilisons pour ce travail le Texte massorétique.

4 Marguerat \& Bourquin, Pour lire les récits bibliques, 24. A en croire Serge Wüthrich, "Deux promenades du lecteur croyant dans les bois de la narratologie et d'ailleurs, ' in Le lecteur. Sixième Colloque International du RRENAB, Université Catholique de Louvain, 24-26 mai 2012. Bibliotheca Ephemeridum Theologicarum Lovaniensium CCLXXIII (ed. Régis Burnet, Didier Luciani, Geert Van Oyen ; Leuven : Peeters, 2015), 195 : la compétence de ce type de lecteur est limitée. En effet, « ne participant pas à la construction du récit (puisqu'il est lui-même construit par le texte), il ne peut qu'actualiser des perspectives induites par un autre. » Ce lecteur implicite, construit par le texte et passif, se distingue du lecteur réel qui est « confronté à un récit à l'élaboration duquel il ne participe qu'à proportion de l'étendue de son encyclopédie personnelle et de sa capacité à identifier des caractéristiques narratives. » cf. Wüthrich, “'Deux promenades du lecteur croyant'”, 195-196. 
Samuel lui annonce sa destitution et, sans attendre la fin de son règne, il prépare sa succession en donnant secrètement l'onction à David.

Après le récit de son onction, le narrateur situe immédiatement David à la cour de Saül et il le présente successivement comme musicien, vaillant guerrier, et un orateur parlant avec discernement (1S 16, 8). Sans tarder, Sauil fait de lui son écuyer. C'est lui qui, par la suite, frappe le géant Goliath qui défiait tout Israël. Ses nombreux succès militaires et son enthousiasme font rapidement de lui un vaillant chef de guerre au point qu'il attire la sympathie de Saül. Mais par la suite, Saül va nourrir d'affreux soupçons et même de la jalousie à son endroit.

\section{B JALOUSIE DE SAÜL ENVERS DAVID}

Plusieurs raisons expliquent la jalousie de Saül vis-à-vis de David d'abord la conscience de son rejet comme roi d'Israël. L'annonce de sa destitution suscite en lui la hantise de savoir quel est ce voisin meilleur que lui (1S 15, 28), que YHWH dit qu'il choisirait pour le remplacer. N'ayant aucune réponse officielle, il va vivre désormais dans le soupçon. Ensuite, il va lentement et progressivement deviner que cet homme est David. Plusieurs éléments en effet vont en ce sens : premièrement l'affection de son fils Jonathan pour David au point qu'il se dépouille de son manteau, de ses habits, de son épée, de son arc et de son ceinturon pour les lui remettre (1S 18,4). Ce faisant, Jonathan lui cède symboliquement son droit de succession. Deuxièmement, les réussites militaires de David (1S 18,5), sa victoire sur Goliath et les éloges des femmes d'Israël : tout cela convainc Saül qu'il ne manque plus à David que la royauté (1S 18,5-8). Saül va désormais jalouser David. Il voit dès lors en lui un rival à éliminer. ${ }^{5}$ Saül n'échappe pas à la règle : il a manifestement peur de David et comprend que le Seigneur est avec David et qu'il l'a abandonné (1S 18,12). «L'attitude de Saül lui-même confirme, s'il était besoin, qu'au fond, c'est à présent David qui est le vrai roi. Son opposition croissante - colère (v. 8), jalousie (v. 9), envies sanguinaires (v. 11), peur et éloignement (v. 12-15) - débouche sur des manœuvres pleines de duplicité visant à éliminer David par la main des Philistins

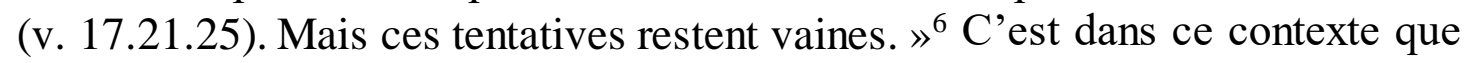
son inquiétude devient grande et sa haine, envers David, définitive (1S 18,29). Devenu gendre du roi, David expérimente l'animosité de son beau-père. Il mesure la gravité du danger qu'il court quand Mikal, sa femme, le fait évader de sa maison par la fenêtre alors que les sbires du roi sont là, prêts à l'arrêter. Mettant judicieusement à profit l'adage salus in fuga, il fuit à Rama où il se

5 Cf. André Wénin, La Bible ou la Violence Surmontée (Paris : Desclée de Brouwer, 2008), 49.

6 Wénin, "David Roi, de Goliath à Bethsabée. La Figure de David dans les Livres de Samuel,' In Louis Desrousseaux, Jacques Vermeylen, Figures de David à travers la Bible. XVIIe Congrès de l'ACFEB - Lille 1-5 septembre 1997 (Cerf : Paris, 1999), 75$112,86$. 
réfugie auprès de Samuel (1S 19,18). Plus tard, informé par Jonathan des intentions meurtrières du roi à son encontre (1S 20,38), il se lance dans une grande cavale qui le fait passer de Nob au désert de Ziph en passant par Qéilla.

\section{DAVID, POURCHASSÉ PAR SAÜL}

David tente donc de fuir Saül qui cherche à le mettre à mort coûte que coûte. «La volonté de Saül d'en finir avec son rival est manifeste dans la promptitude avec laquelle il se met en route pour poursuivre David, et dans le fait qu'il déploie une force proportionnellement égale à celle de l'armée constituée au début de son règne pour combattre les Philistins $(1 \mathrm{~S} 13,2)$, et cinq fois supérieure à la troupe de David $(1 \mathrm{~S} 23,13) . »^{7}$ Sa rage meurtrière devient plus qu'une évidence dès lors qu'il s'en prend non seulement à David, mais aussi à tous ceux qui l'aident : ainsi Saül fait-il passer au fil de l'épée les 85 prêtres de Nob ainsi que tous leurs concitoyens pour ne l'avoir pas averti de la présence de David chez eux et, surtout, pour l'avoir assisté $(1 \mathrm{~S} 22,19)$. Poursuivis par le roi, David et ses hommes n'auraient pas pu lui échapper si un messager n'était venu annoncer à Saül que les Philistins étaient passés à l'attaque (1S 23,27). Au moment de cette alerte, en effet, Saül et sa troupe étaient sur le point d'encercler et de capturer David au désert de Maôn. Cependant, le retour de Saül en Israël n'est que partie remise, puisqu'aussitôt qu'il a écarté le danger philistin, il repart sur les traces de David. Mais vite, les circonstances vont tourner au profit de David qui se trouver par deux fois en position d'éliminer Saül.

\section{RENVERSEMENT DES SITUATIONS : DAVID EPARGNE LA VIE DE SAÜL}

Quand Saül est informé que David se trouve au désert d'Engaddi, il prend 3000 hommes d'élite et se lance à sa poursuite. En 1S 24, la situation s'inverse lorsque Saül se retrouve dans une situation où il peut être tué par David. Celui-ci cependant, alors que ses hommes l'incitent à profiter de cette aubaine, refuse de porter la main sur l'oint de Dieu. (1S 24,7). En effet, il estime que « le roi est un

7 Sophie Ramond, Leçon de Non-Violence pour David : Une Analyse Narrative et Littéraire de 1 Samuel 24-26 (Paris : Cerf, 2007), 34. 
personnage sacré, intouchable $»^{8}$ à telle enseigne que le simple fait de couper furtivement le pan de son manteau ${ }^{9}$ provoque chez lui des palpitations. ${ }^{10}$

David laisse donc Saül sortir intact de la grotte d'Engadi et lorsqu'il s'en éloigne suffisamment, il s'incline jusqu'à terre et se prosterne ${ }^{11}$ avant de lui révéler qu'il a eu l'occasion de l'éliminer, mais ne l'a pas fait. Dans son adresse à Saül, il «oppose sa propre conduite respectueuse à celle de Saül, qui le pourchasse comme s'il était un ennemi dangereux...mais il présente sa décision comme relevant de l'éthique : il a choisi de faire le bien, non le mal alors que le roi ne cesse de le pourchasser. ${ }^{12}$ Et pour persuader Saül de son innocence et de l'absence de méchanceté de sa part, il lui montre le pan du manteau qu'il lui a subrepticement coupé. La réaction de Saül est connue : d'abord, il pleure, ensuite

8 Jacques Vermeylen, “David le Non-Violent,' In La Bible en Récits. L'Exégèse Biblique à l'Heure du Lecteur (ed. Daniel Marguerat ; Genève : Labor et Fides, 2003), 138.

9 Ramond, Leçon de Non-Violence, 37. Couper le pan du manteau est interprété de plusieurs manières : premièrement, le faire serait une manière pour David de s'emparer symboliquement de la royauté. Deuxièmement, empoigner le vêtement d'un supérieur est considéré comme un acte de soumission tandis que lui couper le pan du manteau est une manifestation de révolte, cf. Robert P. Gordon, "David's Rise and Saul's Demise: Narrative Analogy in 1 Samuel 24-26,', Tyndale Bulletin 31 (1980): 56. Troisièmement, à en croire Ramond, le morceau de manteau peut aussi être compris comme une preuve brandie par David pour prouver son innocence, de telle sorte que le pan sert «d'un simple objet-témoin, une preuve volontairement acquise de son innocence », cf. Ramond, Leçon de Non-violence, 39. Par ailleurs, le «pan » (kanap) peut désigner par euphémisme les organes génitaux. Le geste de David revient alors à couper symboliquement les organes génitaux du roi, ce qui pourrait être considéré comme un acte d'élimination de la descendance de Saül, cf. Ramond, Leçon de Nonviolence, 41 (voir Dt 23,1;27,20 ; Lv 18,7 ; Rt 3,9).

10 Selon Fokkelman, ces palpitations sont comme une expression du corps, car en coupant le manteau, David franchit une limite au-delà de laquelle il serait lui-même mort s'il ose porter atteinte à la vie du roi, cf. Jan P. Fokkelman, Narrative Art and Poetry in the Books of Samuel : A Full Interpretation Based on Stylistic and Structural Analyses. Vol. II: The Crossing Fates, 1 Sam. 13-31 \& 2 Sam. 1 (Assen- Dover: NHVan Gorcum, 1986), 457. Néanmoins, le lecteur qui connaît l'histoire peut se demander quel est le degré de sincérité des propos de David. Il est difficile de ne pas soupçonner que ces récits tentent de le présenter comme. En effet, comme l'écrit Keith Whitelam, "The Defence of David,' Journal for the Study of the Old Testament 29 (1984): 79, cette image fortement idéalisée de David aurait été forgée par «la bureaucratie davidique afin de diminuer les suspicions et protéger l'image du roi et de ses successeurs. »

11 La portée de cette prosternation reste ambiguë. Ramond pense que ce geste peut exprimer le respect de David vis-à-vis de Saül, tout comme il peut s'agir d'une astuce pour tromper la vigilance de Saül pour s'attirer toute sa faveur ; cf. Ramond, Leçon de Non-violence, 39.

12 Vermeylen, "David le Non-Violent,"'138. 
il reconnaît que David est plus juste que lui, et enfin il lui demande de jurer qu'il ne fera pas disparaître sa descendance une fois qu'il aura accédé à la royauté (1S 24,17-22).

Curieusement et contre toute attente, lorsque les gens de Ziph informent Saül du lieu où se cache David (1S 26,1), il part à sa recherche. Cette nouvelle poursuite semble aussi décisive que celle relatée en 1S 24. Saül agit, dit Vermeylen, comme s'il ignorait complètement ce qui s'est passé à Engadi, ${ }^{13}$ car l'ardeur de sa fureur contre David n'a pas baissé d'un cran.

Pour sa part, au chapitre 26, David semble aussi plus actif et déterminé qu'il ne l'est au chapitre 24. En effet, tandis que dans l'épisode précédent, il est caché dans une grotte, ici il envoie des espions dans le camp de Saül (1S 26,4) ${ }^{14}$ et descend lui-même dans le campement de Saül.15 Il aurait eu de très bonnes raisons de se laisser aller à la colère et à la vengeance pour en finir une fois pour toutes avec celui qui le traque. Mais c'est plutôt lui qui calme son entourage chaque fois qu'il l'incite à la violence ou à la vengeance. Si en $1 \mathrm{~S} 24$ cet entourage est collectif et anonyme, en 1S 26, il est personnifié par Abishaï, son aide de camp qui lui demande de l'autoriser à clouer Saül à terre avec sa lance d'un seul coup (1S 26,8). Mais David surprend le lecteur en refusant de porter la main sur le roi. ${ }^{16}$ En plus, comme si le refus opposé à Abishaï ne suffisait pas, David ne lui laisse même pas l'occasion d'aller prendre la lance ${ }^{17}$ et la gourde d'eau de Saül comme il le lui demande en $1 \mathrm{~S} 26,11 \mathrm{~b}$ : il la prend lui-même. ${ }^{18}$

13 Vermeylen, "David le Non-Violent," 140. Vermeylen récuse l'idée de considérer ces trois récits comme des variantes d'une même narration. Pour lui, ces récits sont non seulement différents, mais aussi exploités dans des directions différentes même s'ils présentent un schéma commun. La répétition et la succession des éléments présentés dans les trois chapitres auraient donc un effet cumulatif même si chacun des trois récits a sa spécificité.

14 La portée de cet acte est difficile à interpréter, car on se demande si David envoie des émissaires pour savoir où Saül se trouve pour qu'il puisse s'échapper et s'enfuir. Mais la suite du récit n'est pas favorable à pareille interprétation puisque David luimême se rend dans le camp de son adversaire jusqu'à l'endroit où Saül et ses hommes sont couchés. Ce déplacement donne l'impression qu'il veut se livrer à une certaine offensive, même si ce n'est pas le cas, cf. Vermeylen, "'David le Non-Violent,'”140.

15 Il faut noter que c'est la même forme verbale wayyäqom qu' on retrouve aux vv. 2 et 5 pour souligner la détermination qui caractérise désormais David pour aller vers Saül.

16 Ramond, Leçon de Non-Violence, 63.

17 Pour Vermeylen, « la lance qui apparente Saül à Goliath (1S 17,7; 2S 21,19) est donc l'objet qui représente son agressivité brutale à l'égard du fils de Jessé »; Vermeylen, “'David le Non-violent,' 143.

18 A en croire Ramond, David agit précautionneusement parce qu'il n'est pas sûr qu'Abishaï pourra effectivement épargner Saül. Mais le fait de restituer à Saül uniquement sa lance porte une signification : étant donné que la lance est le symbole de 
Ici, par empathie, le lecteur admire la grandeur d'âme de David qui épargne une deuxième fois celui qui ne cesse de le traquer. Comme l'écrit Kahwa, « dans ces deux récits relatifs à la rencontre de David et Saül, l'élément fondamental est l'initiative de David à la recherche de dialogue et de paix avec son bourreau. Premièrement, il parla à Saül après sa sortie de la caverne (1 S 24,9) et deuxièmement, il alla au camp de Saül pour lui parler (1 S 26,5). Il initie les pourparlers en invitant Saül au changement. ${ }^{19}$ Mais pour comprendre le comportement de David en 1S 26, il est intéressant de lire 1S 25. ${ }^{20}$

\section{E LEÇON DE NON-VIOLENCE DONNEE A DAVID EN 1S 25}

Deux personnages font leur entrée dans 1S 25. Il s'agit de Nabal et de sa femme, Abigayil. Cet homme est décrit comme un riche propriétaire. Il compte à son actif mille moutons et mille chèvres. Lorsque David apprend qu'il est en train de tondre ses moutons, il envoie une dizaine de ses serviteurs pour lui adresser une requête en ces termes : «Donne, je te prie, ce que tu peux à tes serviteurs et à ton fils David» (1S 25,8b). David demande en fait d'être récompensé pour avoir protégé ses serviteurs et surtout pour avoir été bienveillant envers eux. Il veut en

force et de violence, en la remettant à Saül, David indique qu'il n'y a pas de violence en lui. En revanche, en gardant la gourde d'eau qui symbolise la vie, il insinue que la vie de Saül touche à sa fin parce qu'il a voulu user de la force et de la violence. Il n'est pas banal de relever que la lance constitue un motif récurrent dans le premier livre de Samuel. En effet, l'usage de la lance par Saül est mentionné 6 fois dans le premier livre de Samuel : il la brandit deux fois contre David $(18,11 ; 19,10)$; une fois contre Jonathan lorsque ce dernier plaide pour David (20,33). Saül est aussi décrit comme ayant une lance à la main quand on lui annonce que les prêtres de Nob ont accueilli David $(22,6)$. Mais la lance est mise en relation avec David quand il la brandit comme trophée $(26,16)$ et lorsqu'il la restitue à Saül $(26,22)$. Malgré cette mise en relation de la lance avec David, il s'agit toujours de la lance de Saül ; Cf. Ramond, Leçon de NonViolence, 63.68.

19 Kahwa Njojo, Ethique de la Non-Violence. Etudes sur Jésus Selon les Evangiles (Genève : Globethics.net, 2013), 193.

20 L'histoire de rédaction de 1S 24 et 26 a fait couler beaucoup d'encre à cause de la ressemblance des incidents qu'ils rapportent. Les similitudes entre les deux récits ont poussé certains exégètes à affirmer qu'ils constituent un doublet rédactionnel rapportant le même événement. Cf. Ralph W. Klein, 1 Samuel (Waco : Word Books Publisher, 1983), 236-237 qui fait un résumé de différents points de vue en rapport avec la datation des textes. D'autres cependant y voient deux récits distincts et considèrent $1 \mathrm{~S} 26$ antérieur à $1 \mathrm{~S}$ 24, ce dernier chapitre étant dérivé du premier et secondaire à lui. Parmi ces exégètes, on peut citer Henry P. Smith, A Critical and Exegetical Commentary on the Books of Samuel. International Critical Commentary (Edinburgh: Clark, 1912), 216, 231; P. K. McCarter, I Samuel: A New Translation with Introduction, Notes and Commentary. Anchor Bible 8 (Garden City : Doubleday, 1984), 375, 386-387. 
fait percevoir « la taxe de fraternité (...) en échange de bons services rendus dans le passé auprès des bergers du riche propriétaire. $»^{21}$

En effet, il était de coutume pour les nomades de tirer des bourgades voisines une taxe pour la protection qu'ils leur avaient accordée, soit en les épargnant du pillage, soit en écartant les brigands. ${ }^{22}$ Qui plus est, David veut mettre toutes les chances de son côté et profiter d'un contexte favorable qu'est la fête de la tonte, une occasion offerte aux propriétaires fortunés de faire preuve de générosité. ${ }^{23}$

Mais Nabal rejette la requête de David. Et comme si cela ne suffisait pas, il tient des propos méprisants à son endroit : «Qui est David, qui est le fils de Jessé ? ...Prendrais-je de mon pain, de mon eau, de ma viande que j'ai abattue pour mes tondeurs, et les donnerai-je à des gens dont je ne sais d'où ils sont?» (1S 25,11). Au rapport que lui font ses serviteurs, David réagit violemment et se met en route avec 400 hommes pour punir Nabal.

Lorsqu'un des serviteurs de Nabal informe Abigayil de la protection qu'ils ont obtenue de David ainsi que de la réponse méprisante que son mari a opposée à la requête de David, elle va à la rencontre de David en apportant avec elle à boire et à manger : 200 pains, deux outres de vin, cinq brebis apprêtées, cinq mesures de blé grillé, cent gâteaux de raisin sec et 200 gâteaux de figues qu'elle fait porter par des serviteurs devant elle (1S 25,18-19).

Arrivée près de David, elle descend rapidement de son âne, tombe sur sa face devant David, se prosterne jusqu'à terre, se jette à ses pieds, et enfin se met à parler (1S 25,23-24). Elle fait « un discours qui est une leçon de sagesse : celle de remettre à Dieu sa propre cause, en évitant de se faire arbitre de la situation. ${ }^{24}$ Ellen von Wolde qualifie ce discours de « tour de force rhétorique $\gg$ qui exerce un effet transformateur sur le futur roi $^{25}$.

Son initiative d'apporter de généreuses provisions à David, et de lui parler pour le dissuader de céder à la violence, finit par sauver non seulement tous les membres de son ménage, mais également d'empêcher le futur roi d'avoir du sang sur ses mains. ${ }^{26}$

Du coup, on réalise combien les propos d'Abigayil sont d'une importance capitale dans le revirement moral de David qui, «malgré la preuve de grande

21 Ramond, Leçon de Non-Violence, 46-47.

22 Ramond, Leçon de Non-Violence, 47.

23 Ramond, Leçon de Non-Violence, 47.

24 Ramond, Leçon de Non-Violence, 52-53.

25 Ellen Von Wolde, «A Leader Led by a Lady: David and Abigail in 1 Samuel 25, »Zeitschrift für die Alttestamentliche Wissenschaft 114 (2002):355-375, 374.

26 Julie Claassens, «An Abigail Optic: Reading the Old Testament at the Intersections, » March 2015; accessed pdf 12/2/2019. 
magnanimité donnée précédemment, lorsqu'il repousse l'occasion de tuer Saül, ${ }^{27}$ marche résolument pour détruire Nabal et tout ce qui lui appartient. En effet, le long discours de la femme empêche David de tirer vengeance de Nabal au moment où « il est complètement pris par son orgueil blessé qui exige justice, par son humiliation qui réclame vengeance. ${ }^{28}$ Abigayil qui voit en David le futur roi, tente de le raisonner : « La stabilité de la future maison royale, dit-elle, est liée à un usage discret de la violence. S'il s'agit de mener les guerres du Seigneur pour la liberté d'Israël, une violence est permise. Elle ne l'est pas en revanche si elle consiste à faire le mal, c'est-à-dire à ceindre l'épée de la vengeance et à tuer à son propre profit (v. 13 ; voir v. 22 et 34$).{ }^{29}$ En d'autres mots, Abigayil signifie à David que, si précédemment, dans le meurtre de Goliath, «l'envie n'était pas le moteur de la violence qu'il avait déployée pour le salut des siens. À présent, se détourner de cette voie, ce serait verser dans le mal et risquer de compromettre gravement sa légitimité de roi désigné par Dieu. Car cela reviendrait à substituer à la loi son seul désir, voire sa seule envie. ${ }^{30}$ On apprendra par la suite que le Seigneur lui-même finit par venger David en frappant Nabal pour son ingratitude. C'est la leçon de non-violence, de pardon et de pitié qui aide David à ne pas punir Nabal. David le reconnaît sans ambages car, pour lui, ce sont les gestes rapides d'Abigaïl, en plus de ses paroles persuasives, qui ont sauvé sa famille d'un massacre violent (1S 25, 32-34). ${ }^{31}$ Aussi, comme l'écrit Ramond, c'est grâce à cette leçon d'Abigayil que David aurait retenu sa violence au moment où une deuxième occasion lui est donnée pour se débarrasser de Saül. En effet, si dans 1 S 24 David arrache symboliquement la royauté à Saül en coupant le pan de son manteau - ce qui est déjà un acte violent - et si dans $1 \mathrm{~S} 25$ il retient sa violence devant Nabal, il semble que l'intervention d'Abigayil a joué un rôle considérable dans le revirement moral de David dans sa deuxième rencontre avec Saül en 1 S $26 .{ }^{32}$

\footnotetext{
27 Ramond, Leçon de Non-Violence, 53.

28 Ramond, Leçon de Non-Violence, 53.

29 André Wénin, « David Roi, de Goliath à Bethsabée. La Figure de David dans les Livres de Samuel, » In Louis Desrousseaux, Jacques Vermeylen, Figures de David à Travers la Bible. XVIIe Congrès de l'ACFEB - Lille 1-5 septembre 1997, Cerf : Paris 1999, p. 75-112, 27.

30 Wénin, « David roi », 27.

31 Claassens, « An Abigail Optic », 5-6.

32 Cf. Ramond, Leçon de Non-violence, 53. Il ne fait l'ombre d'aucun doute que ce récit présente tendancieusement un David non-violent qui maîtrise sa convoitise et sa violence. Comme 1'écrit Steven L. McKenzie, « Who Was King David ? » in Word \& World 23/4 (2003), 357-364; 361-362, les auteurs bibliques s'efforcent de nier l'implication de David dans la mort de Saül en démontrant que David n'a pas tué Saül quand il en a eu l'occasion (1 Sam 24, 26), que la mort de Saül a été ordonnée par Dieu (1 Sam 28), et que David était loin du champ de bataille quand Saül est mort (1 Sam 29-31). Raison pour laquelle il exécute l'homme qui prétend avoir achevé Saül (2 Sam 1). Toutefois, c'est l'acharnement à défendre David qui l'implique dans tous les
} 
Au terme de la lecture de ces trois récits, le lecteur se demande sûrement pour quelle raison David se comporte de cette manière. Aurait-il d'autres raisons d'épargner Saül ? Tâchons de les examiner à présent.

\section{F POUR QUEL MOTIF DAVID ÉPARGNE-T-IL SAÜL EN 1S 24 ET 26 ?}

Selon le texte massorétique, plusieurs raisons peuvent être avancées pour justifier le comportement de David vis-à-vis de Saül. La première serait qu'il a totalement confiance en Dieu à qui il remet sa cause, lui laissant le soin de venger ses ennemis. Il sait qu'il ne lui appartient pas de se venger lui-même - Abigayil le lui a aussi rappelé - puisque la justice comme la vengeance appartiennent à Dieu (1S 24,13.16 ; 26,23-24 ; Dt 32,35 ; Pr 20,22 ; Jr 11,20 ; Rm 12,19). Lui seul s'en charge comme il veut et quand il veut.

La deuxième raison est que David épargne Saül parce que celui-ci est 1'oint de YHWH (1S 24,7.11 ; 26,9). L'expression 'l'oint du Seigneur' (יחשיח יהוה (י) est reprise par David 3 fois en 1S 24 et 3 fois en 1S 26. La triple mention de cette expression dans les deux récits insinue que, pour David, Saül demeure marqué de l'onction de Dieu, et quoi qu'il fasse, il est intouchable. Cette intangibilité du roi semble une tradition bien connue. ${ }^{33}$

Aussi David refuse-t-il de porter la main sur Saül parce qu'il sait qu'en le tuant ou en laissant quelqu'un le tuer, on ne reste jamais impuni. ${ }^{34}$

La troisième raison serait probablement liée au respect de la vie. Saül semble l'admettre lui-même lorsqu'il déclare à David : «je ne te ferai plus de mal, puisque ma vie a eu aujourd'hui tant de prix à tes yeux » $(1 \mathrm{~S} 26,21)$. En des termes presque semblables, David le dit aussi dans sa réplique à Saül : « comme ta vie, aujourd'hui, a eu tant de prix pour moi, que ma vie en ait autant pour le Seigneur » (1S 26,24).

meurtres qu'il semble désapprouver, puisque comme l'écritbien Wénin, quand David fait tuer ceux qui veulent se faire remarquer en tuant ses adversaires potentiels, il savoure entre temps sa « secrète satisfaction ... de voir les choses s'arranger sans qu'il ait à tremper ses mains dans le sang »; cf. Wénin, « David roi », 26 note 77.

33 On le voit, même lorsque Saül demande à son écuyer de tirer sur lui une épée de peur qu'il ne périsse des mains des incirconcis, ce dernier refuse de le faire, car il a peur de mettre fin à la vie d'un roi $(1 \mathrm{~S} 31,4)$. C'est en ce sens que David reproche à l'Amalécite de n'avoir pas craint d'étendre la main pour faire périr Saül même s'il gisait par terre $(2 \mathrm{~S} 1,14)$. L'intangibilité des oints du Seigneur est largement attestée dans la Bible : 2S 1,14.16; 1Ch 16,22; Ps 105,15.

34 Cf. David M. Gunn, The Fate of King Saul: An Interpretation of a Biblical Story (Sheffield: JSOT Press, 1980), 102-103. Dans ce sens, on comprend dorénavant que Saül ne restera pas impuni puisqu'il a attenté à la vie de David, l'oint de YHWH. 
D'aucuns estiment que ce n'est pas par magnanimité que David agit avec une telle prévenance envers Saül, mais que cette attitude participerait plutôt d'une stratégie politique, car « David sait que, si Saül est tué par un rival aspirant à le remplacer, tout roi israélite pourra être tué à son tour. Qu'il ait ou non des visées sur le trône (...), son refus de nuire à Saül a valeur d'investissement dans la stabilité du futur régime - de tout futur régime. ${ }^{35}$

La quatrième hypothèse est que David ne fait aucun mal à Saül parce qu'il le considère comme un frère. Cette idée peut paraître osée ou simpliste puisque Saül et David appartiennent respectivement à la tribu de Benjamin et celle de Juda. Mais, nous parlons de 'fraternité'36, dans son sens le plus large possible, « comme l'attitude par laquelle chacun s'engage à ne pas nuire à autrui (...), comme un ordre social dans lequel chacun aimerait l'autre comme son propre

35 Joel Rosenberg, “'Samuel 1 et 2 : le Jeune David,' In Encyclopédie Littéraire de la Bible (ed. Robert Alter and Frank Kermode ; Paris : Bayard, 2003), 171-172. Fred Catherwood, David, Poète-Guerrier-Roi (Cléon d'Andran- Melhouse : Excelsis - Grâce et Vérité, 2002), 53, abonde dans le même sens : "L'extraordinaire maîtrise dont il (David) avait fait preuve lorsqu'il avait affronté Saül, avait laissé place à la colère et au désir de vengeance. Il avait rappelé à Saül qu'étant l'oint du Seigneur, il était indigne de lui de le pourchasser, mais lui aussi était l'oint du Seigneur, et il n'était certainement pas moins indigne de lui de chercher à se venger d'un éleveur de moutons hargneux. » 36 Le mot "fraternité" est un terme complexe. Il « fait partie de ces termes qui renvoient d'abord à un sens étroit et précis, puis s'étendent ensuite à de multiples significations, en apparence de plus en plus éloignées de la définition originale. Au sens le plus littéral, la fraternité désigne le lien de parenté entre des enfants qui ont les mêmes parents. Au-delà du lien génétique, ce sens du mot 'fraternité' peut s'étendre à la relation affective éprouvée entre frères et sœurs, même issus de parents différents. Dès que l'on ouvre le champ de l'analogie, de multiples déclinaisons du mot 'fraternité' se font jour. Il peut s'agir d'une façon de désigner un lien étroit d'amitié entre deux personnes qui ne sont ni frères ni sœurs ", cf. Alain Vulbeau, "Contrepoint - Le Sens de la Fraternité : Informations Sociales," 93-93. Cited 27 June 2018. Online: http://www.cairn.info/revue-informations-sociales-2012-5-page-93.htm. Dans ce sens, ce terme doit être perçu comme le lien naturel ainsi que le sentiment d'amitié et de solidarité qui allient ou devraient souder tous les hommes constitués désormais comme membres d'une même famille ou espèce humaine. Elle implique la tolérance et le respect mutuel des différences, contribuant ainsi à la paix. Dans ce sens, la fraternité est une valeur de l'humanité comme le stipule l'article premier de la déclaration universelle des droits de l'homme qui énonce :« Tous les êtres humains naissent libres et égaux en dignité et en droits. Ils sont doués de raison et de conscience et doivent agir les uns envers les autres dans un esprit de fraternité » (Déclaration universelle des droits de l'homme). 
frère. ${ }^{37}$ Cette fraternité se concrétise par le sentiment de pitié ${ }^{38}$ éprouvé par David qui ne veut pas faire de mal à Saül, et le traite plutôt avec compassion (1S 24,11).

A y regarder de près, cette hypothèse nous semble plus plausible. En effet, pour la Bible, «si l'homme ne se sent pas responsable envers son frère, la responsabilité et l'humanité se perdent. $"{ }^{39}$ C'est parce que David se sent responsable de l'intégrité et de la vie de Saül qu'il l'épargne et ose condamner Abner pour n'avoir pas veillé sur le roi pendant son sommeil (1S 26,15). La fraternité va donc de pair avec l'humanité et la responsabilité. Peut-on affirmer que la réaction de David a eu un quelconque impact sur l'attitude de Saül ou de Nabal?

\section{G NON-VENGEANCE, UN PAS VERS LA RÉCONCILIATION}

En 1S 24 et 26, le narrateur s'attarde sur l'attitude de Saül quand David lui annonce qu'il l'a regardé avec compassion. Saül n'en croit pas ses yeux. Il demande si c'est bien David qui lui parle. Cette interrogation constitue une remise en question personnelle de Saül qui se rend compte de sa méchanceté visà-vis de David. Raison pour laquelle, s'étant rendu à l'évidence, «il éclate en sanglots, reconnaît que l'accusation de David est fondée, que celui-ci est dans son droit. Il confesse que le mal est de son côté (...) Il admet avoir la preuve de la magnanimité de celui-ci. ${ }^{40}$ Il décide illico de retourner chez lui après avoir prononcé des paroles de bénédiction en faveur de David (1S 26,25).

A en croire Ramond, l'on a à faire ici à une intrigue de révélation. Car Saül apprend d'abord ce qui s'est passé dans la grotte d'Engadi et la manière

37 Jacques Attali, Fraternités : Une Nouvelle Utopie (Paris : Fayard, 1999), 124. Nous n'ignorons pas que cette fraternité est fréquemment mal vécue même parmi les frères consanguins entre lesquels la concurrence, l'envie, la jalousie et la dispute sont souvent au rendez-vous comme le suggèrent les récits de Caïn et Abel, Jacob et Esaü, Joseph et ses frères.

38 Dans 1S 24,11, David dit à Saül : ותחס עליך (et elle a eu pitié de toi). Le verbe חוס "' signifie “'regarder avec compassion", “'avoir pitié”, '“épargner'. Puisqu'il s'agit d'un sentiment intérieur, on peut traduire «mon âme a eu pitié de toi. » Selon Siegfried Wagner, "חוּס "chûs," in Theological Dictionary of the Old Testament. Vol. IV (ed. G. Johannes Botterweck and Helmer Ringgren ; Michigan : Grand Rapids, 1980), 271277, 272, חוס appartient au groupe des verbes décrivant les émotions. Il désigne spécifiquement la sympathie, c'est-à-dire le sentiment de commisération dû à la souffrance, au malheur ou encore au désastre dans lequel quelqu'un est tombé. Dans le cas précis, il s'agit de la pitié que David nourrit à l'endroit de Saül, au point qu'il renonce totalement à le tuer alors qu'il en a l'occasion.

39 AntonVan der Lingen, La Guerre et la Violence dans la Bible (Paris : Cerf, 2016), 70 .

40 Ramond, Leçon de Non-Violence, 40. 
dont David interprète l'événement ${ }^{41}$; ensuite, il est informé et constate que David a pris réellement sa lance et sa gourde d'eau, sans lui faire du mal, alors qu'il était endormi. En effet, fort de cette révélation, Saül cherche à se réconcilier avec David et lui promet de ne plus lui faire du mal $(1 \mathrm{~S} 26,21) .{ }^{42}$ La suite du récit montre que Saül tient parole, car il ne poursuit plus David, même si ce dernier continue à se méfier de lui. ${ }^{43}$

Dans le cas de Nabal, lorsqu'il apprend la colère de David, sa décision de le détruire et l'intervention de sa femme Abigayil sans laquelle il serait déjà détruit, « son cœur mourut dans sa poitrine et il devient comme une pierre » (1S $25,37 b)$.

De ce qui précède, il ressort que l'on peut se servir de la bonté pour chasser le mal de la vie de ceux qui en sont prisonniers. Châtier un ennemi durcirait probablement son cœur tandis que le traiter avec bonté peut l'adoucir. Combattre la jalousie par la jalousie ou la violence par la violence est combattre un incendie avec de la gazoline. En fait, de même qu' " une réponse douce calme la fureur, de même une parole dure excite la colère » $(\operatorname{Pr} 15,1)$. A notre avis, David semble l'avoir compris et c'est pour cela qu'il ne veut pas tuer Saül.

Dans ce sens, selon Vermeylen, même la descente de David dans le camp de Saül n'a d'autre but que de le désarmer moralement, l'emmenant ainsi dans la voie de la non-violence ${ }^{44}$ et de la non-vengeance. Il n'est pas banal de souligner que la loi contre la vengeance de ses ennemis est inscrite comme une règle d'or dans la Bible ${ }^{45}$, même si l'observer n'est pas du tout aisé dès lors que la riposte est le réflexe le plus spontané. Cette séquence narrative indique que la

\footnotetext{
41 Cf. Ramond, Leçon de Non-Violence, 38.

42 Mais on n'est pas certain de la véracité des propos de Saül au vu de tout ce qui s'est passé entre lui et David. A en croire Ramond, cet appel à 'revenir' " peut avoir l'accent d'une menace, car Saül peut se rendre compte qu'il a échoué jusqu'à présent dans sa poursuite de David et qu'il lui faut imaginer un autre plan », Ramond, Leçon de NonViolence, 67.

43 Vermeylen, "David le Non-Violent,"'143.

44 Vermeylen, "David le Non-Violent,"'143.

45 L'Ancien et le Nouveau Testaments fourmillent de passages prescrivant l'amour des ennemis : "Ne dis pas : Je lui ferai comme il m'a fait, Je rendrai à chacun selon ses œuvres" (Pr 24, 29) ; "Si tu rencontres le bœuf de ton ennemi ou son âne égaré, tu le lui ramèneras" (Ex 23,4); "Tu ne te vengeras point, et tu ne garderas point de rancune contre les enfants de ton peuple. Tu aimeras ton prochain comme toi-même. Je suis l'Éternel” (Lv 19, 18) ; «Vous avez entendu qu'il a été dit : Tu aimeras ton prochain et tu haïras ton ennemi. Eh bien, moi, je vous dis : Aimez vos ennemis, et priez pour vos persécuteurs... » (Mt 5, 43-48); «Mais aimez vos ennemis, et faites-leur du bien, et prêtez sans en rien espérer; et votre récompense sera grande, et vous serez les fils du Très-haut ; car il est bon envers les ingrats et les méchants » $(\mathrm{Lc} 6,35)$; «Si ton ennemi a faim, donne-lui à manger ; s'il a soif, donne-lui à boire » $(\mathrm{Rm} 12,20)$.
} 
pitié et la non-violence sont encore possibles. Elles s'enracinent dans le respect de la vie que chaque homme partage avec ses semblables. Le mérite de David est de les avoir cultivées (1S 24, 22-23).

\section{H MA VIE COMPTERA POUR TOI : UN PAS VERS LA PITIE, DEUX PAS VERS LA NON-VIOLENCE}

Dans le dialogue entre David et Saül, deux termes sont mentionnés : 'mon père' (אבי) en 1S 24,12; et 'mon fils' (בני) en 1S 24,17 ; 2S 26,17.21.25. L'usage de ces termes insinue que les deux adversaires se situent au niveau des relations interpersonnelles, de père à fils. Dans ce sens, l'attention peut être focalisée sur la relation entre David et Saül qui se considèrent respectivement 'fils et père'. Ces deux termes ne sont pas à prendre dans leur sens ordinaire. En effet, Saül n'est pas le père géniteur de David, ni ce dernier le fils biologique de Saül. Il est toutefois frappant que David n'appelle Saül 'mon père' qu'une fois en 1S 24 alors que dans la suite du récit, il parle de lui soit comme 'oint du Seigneur', soit comme 'roi'. Quant à Saül, il appelle David 'mon fils' quatre fois $(1 \mathrm{~S} 24,17 ; 2 \mathrm{~S}$ 26,17.21.25).

Dans la requête que David adresse à Nabal par l'intermédiaire de ses serviteurs, il se présente à lui comme son 'fils' : 'Donne ce que tu peux à tes serviteurs et à ton fils David» (1S 25,8c). Nabal refuse de considérer David comme son 'fils', encore moins comme son serviteur. Dans sa réponse arrogante, il s'interroge : « qui est David, qui est le fils de Jessé ? », indiquant par-là qu'il rejette cette paternité, et par voie de conséquence, il dénie la pitié, l'entraide et la compassion aux autres. Pour cela, il renvoie David à l'unique filiation biologique en faisant remarquer que seul Jessé est son père. Pourtant, métaphoriquement parlant, on peut appeler 'frère' toute personne très proche, ${ }^{46}$ et ce terme comporte une nuance affective qui vise à provoquer chez celui qui l'entend le sentiment d'appartenir à vraie famille. ${ }^{47}$

Ainsi, les concepts 'père, fils' utilisés dans cette séquence narrative, débordent le cadre familial restreint et invitent à l'affermissement de nouvelles relations calquées sur celle qui unit chacun à ses propres membres de famille.

46 C'est le cas de David qui considère Jonathan comme son 'frère' $(2 \mathrm{~S} 1,26)$ ou encore le cas du vieux prophète qui enterre l'homme de Dieu qui avait été tué par un lion $(2 \mathrm{R} 13,30)$.

47 Cf. Jan N. Bremmer, "Brothers and Fratricide in the Ancient Mediterranean Israel, Greece and Rome," in Eve's Children. The Biblical Stories Retold and Interpreted in Jewish and Christian Traditions (ed. Gerard F. Luttikhuizen ; Leiden : Brill, 2003), 78. On peut lire avec intérêt Wayne A. Meeks, The First Urban Christians : The Social World of the Apostle Paul, 2nd ed. (New Haven: Yale University Press, 1983), 86-89; Guerrie F. Snyman, “A Hermeneutic of Vulnerability: Redeeming Cain?", in Stellenbosch Theological Journal 2015, Vol 1, No 2, 633-665, http://dx.doi.org /10.17570/stj.2015.v1n2.a30. Consultation du 6/2/2019. 
On pourrait ainsi élargir progressivement le champ, d'une part de la famille restreinte à la famille étendue, d'autre part, de la famille étendue à la famille universelle. C'est à ce niveau précis que peut être évoquée la notion de fraternité universelle. Car «l'enjeu ultime de la fraternité, c'est la vie. ${ }^{48}$

Dès lors, les termes 'père/fils' utilisés dans ces récits sont chargés d'une force rhétorique. D'une part, Saül appelle David 'mon fils' alors qu'il le poursuit pour le mettre à mort ; Nabal méprise David qui se présente à lui comme un 'fils' ; et Saül que David appelle 'père' est celui à qui le 'fils' ne cesse de vouloir échapper. D'autre part, quel fils fuirait devant son père pour se réfugier jusque dans les déserts ? Et quel père oserait prendre 3000 soldats pour pourchasser son fils ? Et pour le cas de Nabal, quel fils peut marcher avec 400 hommes, ceints de leur épée, pour aller détruire la vie de son père ?

C'est ce qui porte à croire que $1 \mathrm{~S}$ 24-26 enseignent la pitié et la nonviolence dans les relations interpersonnelles où la violence fait toujours surface. En effet, le récit de fratricide mentionné en Gn 4 démontre la fragilité des relations fraternelles et la perte d'un monde sans violence. ${ }^{49}$

Il va sans dire que, même si « la violence ne s'arrête pas aux portes de la famille, ${ }^{50}$ ses antidotes, à savoir la non-violence et la pitié en conséquence, sont à cultiver, non plus au paradis, mais dans un monde réel où l'échec inexplicable, l'envie, la jalousie, la haine, la rivalité sont au rendez-vous. ${ }^{51}$ En effet, plus on cultive la pitié, plus mieux on maîtrise la violence et on l'endigue.

\section{FAUT-IL CONCLURE ?}

Certes, il le faut et, surtout en rappelant qu'aucun conflit n'est insurmontable. On le voit en 1S 24-26 où David se trouve entre deux tyrans. D'un côté Saül qui, poussé par une jalousie maladive, le pourchasse pour le mettre à mort. Curieusement, la situation s'inverse à son profit jusqu'à avoir deux occasions tuer Saül, mais il lui épargne la vie. Il profite de cette aubaine pour lui prouver son innocence et lui montrer sa pitié. Fort ému d'expérimenter la générosité de David qui lui 'rend le bien pour le mal' alors que lui ne lui rend que 'le mal pour le bien', Saül n'en croit pas ses yeux, avoue sa faute, reconnaît la justice et l'innocence de David, élève la voix pour pleurer, et promet de ne plus lui faire du mal. De l'autre côté, il y a Nabal, un homme riche, fort important, dur et malfaisant, qui avec une brutalité langagière, méprise David devant ses émissaires et lui refuse des vivres qu'il lui demande pour des services rendus par

\footnotetext{
48 Wénin, La Bible ou la Violence Surmontée, 90.

49 Cf. Bremmer, "Brothers and Fratricide," 91.

50 Ed Noort, "Genesis 4:1-16. From Paradise to Reality: The Myth of Brotherhood," in Eve's Children. The Biblical Stories Retold and Interpreted in Jewish and Christian Traditions. (ed. Gerard F. Luttikhuizen; Leiden: Brill, 2003), 93-106.

51 Cf. Noort, "Genesis 4:1-16,"105.
} 
ses hommes. On observe quasiment le même revirement chez David en 1S 25 : il bénit le Seigneur pour Abigayil, pour son bon sens et pour l'avoir retenu d'en venir au meurtre, prend de la main de celle-ci ce qu'elle avait apporté et lui demande de retourner chez elle en paix (vv.32.33.35). Une lecture attentive de 1S 25,35 ainsi que des derniers versets de $1 \mathrm{~S} 24$ et $26^{52}$ suggèrent un dénouement heureux de l'intrigue de chaque récit: d'une part, malgré sa rage meurtrière, David a pitié de Saül et réussit à vaincre sa soif de vengeance contre Nabal en mettant en pratique les conseils d'Abigayil. D'autre part, Saül parvient à prendre conscience de sa culpabilité, de son péché, de sa folie et de son erreur, ${ }^{53}$ et décide de ne plus faire de mal à David parce que ce dernier a eu pitié de lui $(1 \mathrm{~S} 26,21)$.

Il appert que la vengeance n'est pas invincible, ni la non-violence et la pitié impossibles à cultiver. En effet, au-delà de la violence, il n'y a que la violence et rien ne peut arrêter son cours si ce n'est le refus de se venger. S'il est admis que « la violence homicide se retourne contre son auteur, ${ }^{54}$ il est nécessaire de la limiter, mieux de l'éviter pour pacifier les relations interpersonnelles.

De ce qui précède, on voit combien la non-violence et la pitié peuvent atténuer un conflit. L'apaisement de celui-ci n'est certes pas synonyme de résolution totale. Dans cette séquence narrative, on sait d'une part que, malgré la promesse ferme de Saül à David de ne plus attenter à sa vie, et d'autre part, malgré l'adresse d'Abigayil à son endroit, David n'a rejoint ni le camp de Saül ni la maison de Nabal, ce qui fait dire à Klein « qu'aucune réconciliation durable n'a été réalisée ici. ${ }^{55}$ Mais $M$. Rose pense que cette séparation peut être interprétée de deux manières. Soit qu'elle marque le refus d'admettre qu'il y a eu véritablement réconciliation entre les parties en conflit insinuant que celui-ci persiste; soit elle symbolise le rétablissement de relations harmonieuses dont la distance géographique serait le garant ${ }^{56}$. Comme le fait remarquer A. Wénin, il ne faut pas perdre de vue que « la fraternité fait rarement bon ménage avec la

52 Dans 1S 24, 23, lorsque David jure à Saül de ne pas exterminer sa descendance, ce dernier rentra chez lui et les hommes de David remontèrent au Refuge ; dans $1 \mathrm{~S} 25$, 35 ; lorsque David reçut de la main d'Abigayil ce qu'elle lui avait apporté, David lui dit : Remonte chez toi en paix ; dans 1S 26, 25, Saül dit à David : béni sois-tu mon fils David. Certainement tu entreprendras et tu réussiras. David alla son chemin et Saül retourna chez lui.

53 Ralph W. Klein, 1 Samuel (Waco: Word Books Publisher, 1983), 260.

54 Vermeylen, “David le Non-Violent,'”139. Un lien peut être établi entre la nonvengeance de David et l'amour des ennemis prêché par Jésus (Mt 5, 44 ; Lc 6, 27-28). Abondant dans le même sens, Paul affirme aussi : « Ne répondez jamais au mal par le mal. Cherchez au contraire à faire ce qui est bien devant tous les hommes » (Rm 12, 17).

55 Klein, 1 Samuel, 260.

56 Martin Rose, Une Herméneutique de l'Ancien Testament : Comprendre -Se Comprendre -Faire Comprendre (Genève : Labor et Fides, 2003), 332-333. 
promiscuité » au regard des récits de la Genèse qui montrent des frères se séparer les uns des autres après s'être réconciliés. ${ }^{57}$ En renonçant à la violence, David franchit un pas vers la non-vengeance et cultive la pitié qui, toutes deux, demeurent un véritable défi dans un monde où vengeance, discrimination, guerres, conflits, haine et terrorismes s'érigent en maître.

\section{BIBLIOGRAPHIE SELECTIVE}

Attali, Jacques. Fraternités : Une Nouvelle Utopie. Paris: Fayard, 1999.

Bremmer, Jan N. "Brothers and Fratricide in the Ancient Mediterranean Israel, Greece and Rome." Pages 77-92 in Eve's Children. The Biblical Stories Retold and Interpreted in Jewish and Christian Traditions. Edited by Gerard F. Luttikhuizen, Leiden: Brill, 2003.

Caquot, André \& de Robert, Philippe. Les Livres de Samuel. Commentaire de l'Ancien Testament VI. Genève: Labor et Fides, 1994.

Catherwood, Fred. David, Poète-Guerrier-Roi. Cléon d'Andran- Melhouse : ExcelsisGrâce et Vérité, 2002.

Claassens, Julie. "An Abigail Optic: Reading the Old Testament at the Intersections." March 2015. pdf cited 12 February 2019.

Gordon, Robert P. 'David's Rise and Saul's Demise: Narrative Analogy in 1 Samuel 24 -26,' 'Tyndale Bulletin 31 (1980): 37-64.

Gordon, Robert P. 1 and 2 Samuel. Exeter: Paternoster, 1986.

Gunn, David M. The Fate of King Saul: An Interpretation of a Biblical Story. Sheffield: JSOT Press, 1980.

Fokkelman, Jan P. Narrative Art and Poetry in the Books of Samuel: A Full Interpretation Based on Stylistic and Structural Analyses. Vol. II: The Crossing Fates (I Sam. 13-31 \& II Sam. 1. Studia Semitica Neerlandica 23. Assen-Dover : NH-Van Gorcum, 1986.

Kallemeyn, Harold. "Folie, Violence et Vengeance en 1 Samuel 24-26.' No pages. Cited 15 February 2019. Online: http://larevuereformee.net/ consultation du $15 / 2 / 2019$.

Kahwa Njojo. Ethique de la Non-Violence. Etudes sur Jésus Selon les Evangiles. Genève: Globethics.net, 2013.

Klein, Ralph W. 1 Samuel. World Biblical Commentary 10. Waco : Word Books Publisher, 1983.

57 Wénin, La Bible ou la Violence Surmontée, 27. Lire à ce sujet Gn 33, 16-17; 36, 6-7. Dans le même registre, on peut mettre la réconciliation entre Abraham et Loth après la dispute qui éclate entre leurs pasteurs (Gn 13,7-8) et le traité conclu entre Laban et Jacob (Gn 31, 44-54). En effet, de même que ces deux derniers se séparent après la conclusion du traité (Gn 32,1-2), de même Abraham et Loth vont chacun leur chemin après avoir réglé leur querelle (Gn 13, 11-12). Ce qui fait dire à Kahwa, Ethique de la Non-Violence, 182 que « la séparation non liée à la haine ou à l'envie et au désir égoïste, est un moyen non-violent pour prévenir les violences. Il convient de noter que l'unité résultant de la réconciliation, fruit du pardon, ne veut pas nécessairement dire rester ensemble. On peut être uni tout en étant séparé géographiquement. » 


\section{Kagunge, “David versus Saül et Nabal,” OTE 32/1 (2019): 155-173}

McCarter, P. K. I Samuel: A New Translation with Introduction, Notes and Commentary. Anchor Bible 8 (Garden City: Doubleday, 1984).

McKenzie, Steven L. "Who Was King David ?" Word \& World 23/4 (2003): 357-364.

Meeks, Wayne A. The First Urban Christians: The Social World of the Apostle Paul, 2nd ed. New Haven: Yale University Press, 1983.

Patrick D. Miller, Patrick D. \& Robert, J.J.M. The Hand of the Lord: A Reassessment of the "Ark Narrative', of 1 Samuel (Baltimore: Johns Hopkins University Press, 1977.

Noort, Ed. "Genesis 4:1-16. From Paradise to Reality: The Myth of Brotherhood." Pages 93-106 in Eve's Children. The Biblical Stories Retold and Interpreted in Jewish and Christian Traditions. Edited by Gerard F. Luttikhui zen, Leiden : Brill, 2003.

Ramond, Sophie. Leçon de Non-Violence pour David: Une Analyse Narrative et Littéraire de 1 Samuel 24-26. Paris : Cerf, 2007.

Rose, Martin. Une Herméneutique de l'Ancien Testament : Comprendre-Se Comprendre -Faire comprendre. Genève : Labor et Fides, 2003.

Smith, Henry P. A Critical and Exegetical Commentary on the Books of Samuel. International Critical Commentary (Edinburgh: Clark, 1912).

Snyman, Gerrie F. "A Hermeneutic of Vulnerability : Redeeming Cain ?" Stellenbosch Theological Journal 1 (2015/2): 633-665. Cited 6 February 2019. DOI: https://doi.org/10.17570/stj.2015.v1n2.a30.

Spinoza, Baruch. Euvres Complètes. Trad. M. Francès. Paris : Bibliothèque de la Pléïade, 1954.

Van der Lingen, Anton. La Guerre et la Violence dans la Bible. Lire la Bible 189. Paris : Cerf, 2016.

Vermeylen, Jacques. “David le Non-Violent,' Pages 135-152 In La Bible en Récits. L'Exégèse Biblique à l'Heure du Lecteur. Edited by Daniel Marguerat, Genève : Labor et Fides, 2003.

Von Wolde, Ellen. "A Leader Led by a Lady: David and Abigail in 1 Samuel 25," Zeitschrift für die Alttestamentliche Wissenschaft 114 (2002): 355-375.

Vulbeau, Alain. "Contrepoint - Le Sens de la Fraternité : Informations Sociales," $93-$ 93. Cited 27 June 2018. Online: http://www.cairn.info/revue-informationssociales-2012-5-page-93.htm.

Wagner, Siegfried. חוּ " 'chûs,' Pages 271-277 in Theological Dictionary of the Old Testament. Vol. IV. Edited by G. Johannes Botterweck and Helmer Ringgren, Michigan: Grand Rapids, 1980.

Wénin, André. La Bible ou la Violence Surmontée. Paris : Desclée de Brouwer, 2008.

Wénin, André. "David Roi, de Goliath à Bethsabée. La Figure de David dans les Livres de Samuel. " Pages 75-112 In Louis Desrousseaux, Jacques Vermeylen, Figures de David à travers la Bible. XVIIe Congrès de l'ACFEB - Lille 1-5 septembre 1997. Cerf : Paris, 1999, 75-112.

Whitelam, Keith. 'The Defense of David,' Journal for the Study of the Old Testament 29 (1984): 61-87. https://doi.org/10.1177/030908928400902904.

Wüthrich, Serge. "Deux Promenades du Lecteur Croyant dans les Bois de la Narratologie et d'Ailleurs. » Pages 193-208 in Le lecteur. Sixième Colloque International du RRENAB, Université Catholique de Louvain, 24-26 mai 2012. Bibliotheca Ephemeridum Theologicarum Lovaniensium CCLXXIII. Edited by Régis Burnet, Didier Luciani, Geert Van Oyen, Louvain Peeters, 2015. 
Kagunge, “David versus Saül et Nabal,” OTE 32/1 (2019): 155-173

Mr Augustin Lwamba Kagunge, Katholieke Universteit, Leuven, Belgium. Email: augustin.lwambakagunge @ student.kuleuven.be; augustin.lwamba @ucc.ac.cd; ORCID: https://orcid.org/0000-0002-5659-9513. 